\title{
GENERIC LIE COLOUR ALGEBRAS
}

\author{
Kenneth L. Price
}

\begin{abstract}
We describe a type of Lie colour algebra, which we call generic, whose universal enveloping algebra is a domain with finite global dimension. Moreover, it is an iterated Ore extension. We provide an application and show Gröbner basis methods can be used to study universal enveloping algebras of factors of generic Lie colour algebras.
\end{abstract}

\section{INTRODUCTION}

Throughout $k$ denotes a field of characteristic different from 2. Iterated Ore extensions play an important role in this work. All of the Ore extensions we introduce are of the form $A[\theta ; \sigma, \delta]$ where $A$ is a $k$-algebra, $\sigma$ is an automorphism, and $\delta$ is a $\sigma$-derivation. In this case if $A$ is Noetherian, a domain, or has finite right global dimension, then $A[\theta ; \sigma, \delta]$ has that corresponding property as well. These facts are well known (see [11, Theorem I.2.9 and Theorem VII.5.3 ]) and will be invoked as needed without further comment.

We follow the notation on Lie colour algebras in [13, Sections 1, 2, and 6]. Let $k^{\times}=k \backslash\{0\}$ denote the group of units of $k$.

Definition 1.1: Let $G$ be an Abelian group. A map $\varepsilon: G \times G \rightarrow k^{\times}$is called a skew-symmetric bicharacter on $G$ if it satisfies (1) and (2) below, for any $f, g, h \in G$.

1. $\varepsilon(f, g+h)=\varepsilon(f, g) \varepsilon(f, h)$ and $\varepsilon(g+h, f)=\varepsilon(g, f) \varepsilon(h, f)$

2. $\varepsilon(g, h) \varepsilon(h, g)=1$

All gradings are with respect to an additively written Abelian group. We denote the degree of a nonzero homogeneous element $x$ by $\partial x$. We want to avoid statements like " $\partial x=g$ or $x=0$ " so we often write $\partial x=g$ to handle both cases. If $x$ and $y$ are homogeneous and $\varepsilon$ is a skew-symmetric bicharacter, then we shorten our notation by writing $\varepsilon(x, y)$ instead of $\varepsilon(\partial x, \partial y)$.

DEFINITION 1.2: A $(G, \varepsilon)$-Lie colour algebra is a $G$-graded vector space $\mathcal{L}$ equipped with a graded bilinear map $\langle\rangle:, \mathcal{L} \times \mathcal{L} \rightarrow \mathcal{L}$, called the bracket of $\mathcal{L}$, which satisfies the following for any homogeneous $x, y, z \in \mathcal{L}$.

$$
\begin{array}{cc}
\langle x, y\rangle=-\varepsilon(x, y)\langle y, x\rangle & \varepsilon \text {-skew-symmetry } \\
\varepsilon(z, x)\langle x,\langle y, z\rangle\rangle+\varepsilon(y, z)\langle z,\langle x, y\rangle)+\varepsilon(x, y)\langle y,\langle z, x\rangle\rangle=0 & \varepsilon \text {-Jacobi identity }
\end{array}
$$

Received 17th November, 2004

Copyright Clearance Centre, Inc. Serial-fee code: 0004-9727/05 \$A2.00+0.00. 
A skew-symmetric bicharacter $\varepsilon$ satisfies $\varepsilon(g, g)= \pm 1$ for each $g \in G$. Note that

$$
G_{+}=\{g \in G: \varepsilon(g, g)=1\}
$$

is a subgroup such that $\left[G: G_{+}\right] \leqslant 2$. Set

$$
G_{-}=\{g \in G: \varepsilon(g, g)=-1\}=G \backslash G_{+} .
$$

For any $G$-graded vector space $V$ we set $V_{ \pm}=\bigoplus_{g \in G_{ \pm}} V_{g}$.

We may view Lie superalgebras and graded Lie algebras, which are graded over $\mathbb{Z}_{2}$ and $\mathbb{Z}$, respectively, as special types of Lie colour algebras. The 'absolutely torsion free' condition was introduced by R. Bøgvad to find graded Lie algebras with finite global dimension (see [5, Theorem 1]). It is well-known that the universal enveloping algebra of a finite dimensional Lie superalgebra may have infinite global dimension (see [3, Proposition 5]). M. Aubry and J.-M. Lemaire have shown that the universal enveloping algebra of an absolutely torsion free graded Lie algebra (or Lie superalgebra) is a domain (see [1]).

Now consider a finite dimensional Lie colour algebra $(\mathcal{L},\langle\rangle$,$) . Its universal enveloping$ algebra $U(\mathcal{L})$ need not even be semiprime (see [13, Example 2.9]). Conditions for $U(\mathcal{L})$ to be semiprime or prime are provided in [13, Theorem 2.5], which extends the analogous result, $[4$, Theorem 1.5], for Lie superalgebras. When $U(\mathcal{L})$ has finite global dimension, then it must equal $\operatorname{dim} \mathcal{L}_{+}$by $[\mathbf{1 2}$, Theorem 3.1], which extends the analogous result, $[\mathbf{9}$, Proposition 2.3], for Lie superalgebras.

We do not know how to extend Aubry and Lemaire's or Bøgvad's theorems to Lie colour algebras (see [1] and [5]). However we believe it may be achieved through the use of generic Lie colour algebras, which are defined in Section 2.

We handle the case with $\operatorname{dim} \mathcal{L}_{-} \leqslant 2$ in Section 3. In this case Theorem 3.5 provides conditions for $U(\mathcal{L})$ to be a domain with finite global dimension. Example 3.2 shows this is possible even when there exists $x \in \mathcal{L}_{-}$such that $\langle x, x\rangle=0$. Results of Behr, Bøgvad, and Aubry and Lemaire (discussed above) imply that this is not possible for Lie superalgebras or graded Lie algebras. Our proof uses generic Lie colour algebras.

In Section 4 we explain how to find the Gröbner basis of an ideal generated by positive elements of a generic Lie colour algebra. We refer the reader to [7] for background on Gröbner bases, to [8] for a Gröbner basis test to determine if an ideal of an iterated Ore extension is completely prime, and to [6] for a Gröbner basis method to calculate projective dimension.

\section{Generic lie colour Algebras}

For a Lie colour algebra $(\mathcal{L},\langle\rangle$,$) and a linear subspace V$ of $\mathcal{L}$ - we let $\langle V, V\rangle$ denote the linear subspace of $\mathcal{L}_{+}$generated by brackets between elements of $V$. If $n=\operatorname{dim} V$ then it is easy to see that $\operatorname{dim}\langle V, V\rangle \leqslant n(n+1) / 2$. 
Definition 2.1: A Lie colour algebra $(\mathcal{X},\langle\rangle$,$) is called generic if \operatorname{dim} \mathcal{X}_{+}$ $=m(m+1) / 2$, where $m=\operatorname{dim} \mathcal{X}_{-}<\infty$, and $\mathcal{X}_{+}=\left\langle\mathcal{X}_{-}, \mathcal{X}_{-}\right\rangle$is colour central, that is, $\langle x, y\rangle=0$ for all $x \in \mathcal{X}_{+}$and $y \in \mathcal{X}$.

REMARK 2.2. Let $V$ be a graded subspace of $\mathcal{X}_{-}$. We set $\mathcal{L}_{-}=V$ and $\mathcal{L}_{+}=\langle V, V\rangle$. Then it is easy to show the sub Lie colour algebra $\mathcal{L}$ of $\mathcal{X}$ is also a generic Lie colour algebra.

Lemma 2.3. Let $(\mathcal{L},\langle\rangle$,$) be a finite dimensional Lie colour algebra such that$ $\mathcal{L}_{+}=\left\langle\mathcal{L}_{-}, \mathcal{L}_{-}\right\rangle$is colour central. Then there is a generic Lie colour algebra $\mathcal{X}$ with $\operatorname{dim} \mathcal{X}_{-}=\operatorname{dim} \mathcal{L}_{-}$and a surjective Lie colour algebra homomorphism $\psi: \mathcal{X} \rightarrow \mathcal{L}$.

The proof of Lemma 2.3 is straightforward.

Given a $G$-graded algebra $A$ we write $A^{\varepsilon}\left[t_{1}, t_{2}, \ldots, t_{n}\right]$ to denote the colour polynomial algebra over $A$ in $n$ homogeneous variables. It is $G$-graded and isomorphic to an iterated Ore extension of $A$ (see [2] for details).

THEOREM 2.4. Suppose $\mathcal{X}$ is generic and $x_{1}, \ldots, x_{m}$ form a homogeneous basis of $\mathcal{X}_{-}$. Set $g_{i}=\partial x_{i}$ and $t_{i, j}=\left\langle x_{i}, x_{j}\right\rangle / 2$ for $1 \leqslant i<j \leqslant n$. Then $U(\mathcal{X})$ is isomorphic to the iterated Ore extension

$$
U(\mathcal{X}) \cong S\left[x_{1} ; \alpha_{1}\right]\left[x_{2} ; \alpha_{2}, \delta_{2}\right] \ldots\left[x_{m} ; \alpha_{m}, \delta_{m}\right]
$$

with $S=k^{\varepsilon}\left[t_{i, j}: 1 \leqslant i<j \leqslant m\right]$ such that for all $a \leqslant m$ and $1 \leqslant b<c \leqslant m$

1. $\alpha_{a}\left(t_{b, c}\right)=\varepsilon\left(g_{a}, g_{b}+g_{c}\right) t_{b, c}$

2. $\delta_{a}\left(t_{b, c}\right)=0$,

3. $\alpha_{c}\left(x_{b}\right)=\varepsilon\left(g_{c}, g_{b}\right) x_{b}$,

4. and $\delta_{c}\left(x_{b}\right)=-2 \varepsilon\left(g_{c}, g_{b}\right) t_{b, c}=\left\langle x_{c}, x_{b}\right\rangle$.

Theorem 2.4 can be proved in the same way as [10, Theorem II.3.1]. In fact the definition of generic Lie colour algebra was motivated by the treatment of generic Clifford algebras in [10, Chapter 2].

\section{An Application}

We assume $k$ is algebraically closed throughout this section.

Lemma 3.1. Let $\left(\mathcal{L}_{,}(\rangle,\right)$be a Lie colour algebra such that $\mathcal{L}_{+}$is colour central and $\operatorname{dim} \mathcal{L}_{-}=\operatorname{dim} \mathcal{L}_{+}=2$. Suppose there does not exist homogeneous $x \in \mathcal{L}_{-}$such that $\langle x, x\rangle=0$. Then $U(\mathcal{L}) \cong k\left[\theta_{1}\right]\left[\theta_{2} ; \sigma_{q}\right]$ where $q$ is a nonzero scalar, $\sigma_{q}\left(\theta_{1}\right)=q \theta_{1}$, and either $\theta_{1}, \theta_{2}$ are homogeneous or $\theta_{1}+\theta_{2}, \theta_{1}-\theta_{2}$ are homogeneous and $q=-1$.

Proof: In view of Lemma 2.3 we may pass to the case that $\mathcal{L} \cong \mathcal{X} / K$ where $K=\operatorname{ker} \psi$ is a homogeneous linear subspace of $\mathcal{X}_{+}$with $\operatorname{dim} K=1$. 
STEP 1. Choose nonzero homogeneous $t \in K$. There is a homogeneous basis $\left\{x_{1}, x_{2}\right\}$ of $\mathcal{X}_{-}$such that either

$$
t=\lambda_{1}\left\langle x_{1}, x_{1}\right\rangle+\lambda_{2}\left\langle x_{2}, x_{2}\right\rangle \text { or } t=\left\langle x_{1}, x_{2}\right\rangle .
$$

Set $g_{1}=\partial x_{1}$ and $g_{2}=\partial x_{2}$.

Let $y_{1}, y_{2}$ form a homogeneous basis of of $\mathcal{X}_{-}$. Then we may write $t$ as in equation 2 for some $\mu_{1}, \mu_{2}, \mu_{3} \in k$.

$$
t=\mu_{1}\left\langle y_{1}, y_{1}\right\rangle+\mu_{2}\left\langle y_{2}, y_{2}\right\rangle+\mu_{3}\left\langle y_{1}, y_{2}\right\rangle
$$

If $\mu_{3}=0$ or $\mu_{1}=\mu_{2}=0$ then step 1 follows immediately. We pass to the case $\mu_{2} \neq 0$ and $\mu_{3} \neq 0$ by relabeling $y_{1}$ and $y_{2}$ if necessary. This implies

$$
\partial t=2 \partial y_{2}=\partial y_{1}+\partial y_{2}
$$

which yields $\partial y_{1}=\partial y_{2}$. Set

$$
\lambda_{1}=\mu_{1}-\left(2^{-1} \mu_{3}\right)^{2}\left(\mu_{2}\right)^{-1}, \quad x_{1}=y_{1}, \quad \lambda_{2}=\mu_{2},
$$

and

$$
x_{2}=y_{2}+\mu_{3}\left(2 \mu_{2}\right)^{-1} y_{1} .
$$

A straightforward calculation shows

$$
t=\lambda_{1}\left\langle x_{1}, x_{1}\right\rangle+\lambda_{2}\left\langle x_{2}, x_{2}\right\rangle .
$$

STEP 2. Set $T=U(\mathcal{X})$. Then $T \cong k\left[t_{12}\right]\left[x_{1} ; \sigma_{1}\right]\left[x_{2} ; \sigma_{2}, \delta_{2}\right]$, defined as in Theorem 2.4, and $U(\mathcal{L}) \cong T /(t)$.

The last statement follows from universal properties of enveloping algebras and Ore extensions.

STEP 3. If $t=\left\langle x_{1}, x_{2}\right\rangle$ the lemma holds with $q=\varepsilon\left(g_{1}, g_{2}\right)$. In this case $\theta_{1}$ and $\theta_{2}$ are homogeneous.

This is easy since $t=2 t_{12}$ so $U(\mathcal{L}) \cong T /\left(t_{12}\right)$.

STEP 4. Suppose $t=\lambda_{1}\left\langle x_{1}, x_{1}\right\rangle+\lambda_{2}\left\langle x_{2}, x_{2}\right\rangle$. The lemma holds with $q=-1$. If $\partial x_{1}=\partial x_{2}$ then $\theta_{1}$ and $\theta_{2}$ are homogeneous. Otherwise $\theta_{1}+\theta_{2}$ and $\theta_{1}-\theta_{2}$ are homogeneous.

If $\lambda_{i}=0$ for $i=1$ or $i=2$ then $\langle x, x\rangle=0$ with homogeneous $x=\psi\left(x_{i}\right) \in \mathcal{L}_{-}$. We assumed this could not happen. Thus $\lambda_{1} \neq 0$ and $\lambda_{2} \neq 0$ which implies $\partial t=2 g_{1}=2 g_{2}$ and $\varepsilon\left(g_{1}, g_{2}\right)^{2}=1$. By replacing $x_{1}$ and $x_{2}$ by appropriate scalar multiples, if necessary, we may assume $\lambda_{1}=1$ and $\lambda_{2}=-1$.

Set $S=k\left[u, z_{1}\right]\left[z_{2} ; \sigma, \delta\right]$, where $\sigma\left(z_{1}\right)=-z_{1}, \sigma(u)=u, \delta\left(z_{1}\right)=2 u$ and $\delta(u)=0$. There is an isomorphism $\phi: T \rightarrow S$ determined by $\phi\left(x_{1}\right)=z_{1}+z_{2}, \phi\left(x_{2}\right)=z_{1}-z_{2}$, and $\phi\left(t_{12}\right)$ is given by equation 3 .

$$
\phi\left(t_{12}\right)=\frac{1}{2}\left(1-\varepsilon\left(g_{1}, g_{2}\right)\right)\left(\left(z_{1}\right)^{2}-\left(z_{2}\right)^{2}\right)+\left(1+\varepsilon\left(g_{1}, g_{2}\right)\right)\left(u-z_{1} z_{2}\right)
$$


Then $\phi(t)=u$ so $U(\mathcal{L}) \cong S /(u)$.

EXAMPLE 3.2. Suppose $\varepsilon$ is a skew-symmetric bicharacter on $G$ and there exist $g_{1}, g_{2}$ $\in G_{-}$such that $2 g_{1}=2 g_{2}$, and $\varepsilon\left(g_{1}, g_{2}\right)=1$ (bicharacters with this property are described in [13, Lemma 2.7]). Let $\mathcal{L}$ be the Lie colour algebra with homogeneous basis $\left\{u_{1}, u_{2}, x_{1}, x_{2}\right\}$ such that $\partial u_{1}=2 g_{1}=2 g_{2}, \partial u_{2}=g_{1}+g_{2}, \partial x_{1}=g_{1}, \partial x_{2}=g_{2}$, and the brackets between basis elements are all zero except for the ones listed below.

$$
\left\langle x_{1}, x_{1}\right\rangle=2 u_{1} \quad\left\langle x_{2}, x_{2}\right\rangle=2 u_{1} \quad\left\langle x_{1}, x_{2}\right\rangle=u_{2} \quad\left\langle x_{2}, x_{1}\right\rangle=-u_{2}
$$

Choose $\zeta \in k$ which satisfies $\zeta^{2}=-1$, then $x=x_{1}+\zeta x_{2}$ satisfies $\langle x, x\rangle=0$. However $U(\mathcal{L}) \cong k\left[\theta_{1}\right]\left[\theta_{2} ; \sigma_{-1}\right]$ by the proof of Lemma 3.1.

REMARK 3.3. The product of linearly independent elements $x_{1}-x_{2}, x_{1}+x_{2} \in \mathcal{L}_{-}$is $u_{2} \in \mathcal{L}_{+}$. At first glance this may appear to violate the PBW theorem (see [2, Theorem 3.2.2]). It does not since the elements $x_{1}-x_{2}$ and $x_{1}+x_{2}$ are not homogeneous.

REMARK 3.4. In example $3.2, \mathcal{L} \cong L^{\gamma}$ for some torsion free Lie superalgebra $L$ with appropriate $G$-grading and $\gamma$ a two-cocycle on $G$ (see [13, Corollary 6.1] for notation). There is an algebra isomorphism $U(\mathcal{L}) \cong U(L)^{\gamma} \cong U(L)$.

Theorem 3.5. Let $(\mathcal{L},\langle\rangle$,$) be a finite dimensional Lie colour algebra. Suppose$ $\operatorname{dim} \mathcal{L}_{-} \leqslant 2$ and $\operatorname{dim} V \leqslant \operatorname{dim}\langle V, V\rangle$ for each graded subspace $V$ of $\mathcal{L}_{-}$. Then $U(\mathcal{L})$ is a domain with global dimension equal to $\operatorname{dim} \mathcal{L}_{+}$.

Proof: We may reduce to the case $\mathcal{L}_{+}$is colour central by [13, Lemma 6.2] and [11, Corollary 6.18]. Let $\mathcal{L}^{\prime}$ be the smallest sub Lie colour algebra which contains $\mathcal{L}_{-}$. Then $\mathcal{L}_{+}^{\prime}=\left\langle\mathcal{L}_{-}, \mathcal{L}_{-}\right\rangle, \mathcal{L}_{-}^{\prime}=\mathcal{L}_{-}$, and $U(\mathcal{L})$ is a colour polynomial algebra over $U\left(\mathcal{L}^{\prime}\right)$. Thus we may pass to the case $\mathcal{L}=\mathcal{L}^{\prime}$. If $\operatorname{dim} \mathcal{L}_{-}^{\prime}=\operatorname{dim} \mathcal{L}_{+}^{\prime}=2$ then we may apply Lemma 3.1. Otherwise apply Lemma 2.4.

\section{GRÖBNER BASIS METHODS}

Throughout this section $\mathcal{X}$ denotes a generic Lie colour algebra and $x_{1}, x_{2}, \ldots, x_{m}$ form a homogeneous basis of $\mathcal{X}_{-}$. We define a chain of generic sub Lie colour algebras which are used to express $U(\mathcal{X})$ as an iterated Ore extension. Then we explain how to find the Gröbner basis of an ideal generated by homogeneous elements of $\mathcal{X}_{+}$.

Set $l=(m-1) / 2 m$ and $p=l+m$. We specify a homogeneous subset of $\mathcal{X}$ and define a function $\phi:\{1,2, \ldots, p\} \rightarrow\{1,2\}$. Fix the following notation for $1 \leqslant i \leqslant j \leqslant m$.

1. $s(i, j)=j(j-1) / 2+i$

2. $t_{s(j, j)}=x_{j}$

3. $\phi(s(j, j))=2$

4. If $i<j$ then $t_{s(i, j)}=\left\langle x_{i}, x_{j}\right\rangle$ and $\phi(s(i, j))=1$. 
For each $\alpha=\left(\alpha_{1}, \alpha_{2}, \ldots, \alpha_{p}\right) \in \mathbb{N}^{p}$ we write $t^{\alpha}=t_{1}^{\alpha_{1}} t_{2}^{\alpha_{2}} \cdots t_{p}^{\alpha_{p}} \in U(\mathcal{X})$. Set $e_{i}=\left(0, \ldots, \frac{1}{(i)}, \ldots, 0\right) \in \mathbb{N}^{p}$ for $1 \leqslant i \leqslant p$. Then a homogeneous basis for $\mathcal{X}_{+}$is

$$
\left\{\left(t_{i}\right)^{\phi(i)}: 1 \leqslant i \leqslant p\right\}=\left\{t^{\phi(i) e_{i}}: 1 \leqslant i \leqslant p\right\} .
$$

Proceeding as in Remark 2.2, we let $\mathcal{X}_{i}$ be the generic sub Lie colour algebra generated by $\left\{x_{1}, x_{2}, \ldots, x_{i}\right\}$.

Lemma 4.1. Set $U_{1}=k\left[t_{1}\right]$, and for $j=2, \ldots, m$, define $U_{j}$ recursively by equation 4.

$$
U_{j}=U_{j-1}\left[t_{s(1, j)} ; \sigma_{s(1, j)}\right] \cdots\left[t_{s(j-1, j)-1} ; \sigma_{s(j-1, j)}\right]\left[t_{s(j, j)} ; \sigma_{s(j, j)}, \delta_{s(j, j)}\right]
$$

1. Then $\mathcal{B}=\left\{\mathbf{t}^{\alpha}: \alpha \in \mathbb{N}^{p}\right\}$ is a basis for $U(\mathcal{X})$,

2. $U\left(\mathcal{X}_{i}\right) \cong U_{i}$ for $1 \leqslant i \leqslant m$, and

3. there is a $\mathcal{B}$-admissible ordering $\preceq$ on $U(\mathcal{X})$ such that $t_{1} \prec t_{2} \prec \cdots \prec t_{p}$.

Proof: Part 1 follows from the PBW Theorem. Part 2 can be proved in the same way as [10, Theorem 3.1]. Part 3 follows from part 2 and [7, Theorem 1.10]. The maps $\sigma_{1}, \sigma_{2}, \ldots, \sigma_{p}$ and $\delta_{3}, \delta_{6}, \ldots, \delta_{p}$ are determined by the rules below.

(i) $\sigma_{j}\left(t_{i}\right)=\varepsilon\left(t_{j}, t_{i}\right) t_{i}$ for $1 \leqslant i<j \leqslant p$

(ii) $\delta_{s(j, j)}\left(t_{i}\right)=\left\langle t_{s(j, j)}, t_{i}\right\rangle$ for $1 \leqslant j \leqslant m$ and $1 \leqslant i<s(j, j)$

We adopt notation and terminology from [7].

Definition 4.2: Choose nonzero $u \in U(\mathcal{X})$. By part 1 of Lemma 4.1 the expression in equation 5 is unique with $c_{\alpha} \in k$ for each $\alpha \in \mathbb{N}^{p}$.

$$
u=\sum_{\alpha \in \mathbb{N}^{p}} c_{\alpha} \mathbf{t}^{\alpha}
$$

1. Equation 5 is called the standard representation of $u$.

2. The Newton diagram of $u$ is $\mathcal{N}(u)=\left\{\alpha \in \mathbb{N}^{p}: c_{\alpha} \neq 0\right\}$.

3. The exponent of $u$ is $\exp (u)=\max _{\preceq} \mathcal{N}(u)$.

4. The leading coefficient of $u$ is $l c(u)=c_{\exp (u)}$.

In particular, if $u \in \mathcal{X}_{+}$then $\mathcal{N}(u) \subseteq\left\{\phi(i) e_{i}: 1 \leqslant i \leqslant p\right\}$.

DEFINITION 4.3: Let $u_{1}, u_{2} \in U(\mathcal{X})$ be given and set

$$
\alpha=\left(\alpha_{1}, \alpha_{2}, \ldots, \alpha_{p}\right)=\exp \left(u_{1}\right)
$$

and

$$
\beta=\left(\beta_{1}, \beta_{2}, \ldots, \beta_{p}\right)=\exp \left(u_{2}\right) .
$$

Let $\gamma=\left(\gamma_{1}, \gamma_{2}, \ldots, \gamma_{p}\right)$ be such that $\gamma_{i}=\max \left\{\alpha_{i}, \beta_{i}\right\}$ for each $i=1,2, \ldots, p$. The left $S$-polynomial of $u_{1}$ and $u_{2}$, denoted $S^{\ell}\left(u_{1}, u_{2}\right)$, is shown in equation 6 where $\lambda=l c\left(u_{2}\right)\left(l c\left(\mathbf{t}^{\alpha} \mathbf{t}^{\gamma-\alpha}\right)\right)^{-1}$ and $\mu=l c\left(u_{1}\right)\left(l c\left(\mathbf{t}^{\beta} \mathbf{t}^{\gamma-\beta}\right)\right)^{-1}$.

$$
S^{\ell}\left(u_{1}, u_{2}\right)=\lambda \mathrm{t}^{\gamma-\alpha} u_{1}-\mu \mathrm{t}^{\gamma-\beta} u_{2}
$$


LEMMA 4.4. If $K$ is a homogeneous linear subspace of $\mathcal{X}_{+}$then there is a homogeneous basis $\mathcal{G}=\left\{u_{1}, u_{2}, \ldots, u_{n}\right\}$ of $K$ such that $u_{1} \prec u_{2} \prec \cdots \prec u_{n}$ and $\exp \left(u_{i}\right) \notin \mathcal{N}\left(u_{j}\right)$ for all $i, j$ with $1 \leqslant i<j \leqslant n$.

Proof: We prove such a basis $\mathcal{G}$ exists by induction on $n$, with the case $n=1$ being trivial.

STEP 1. If $K^{\prime}$ is a homogeneous linear subspace of $K$ with $\operatorname{dim} K^{\prime}=n-1$ then there is a basis $\mathcal{G}^{\prime}=\left\{u_{1}, u_{2}, \ldots, u_{n-1}\right\}$ of $K^{\prime}$ such that $u_{1} \prec u_{2} \prec \cdots \prec u_{n-1}$ and $\exp \left(u_{i}\right) \notin \mathcal{N}\left(u_{j}\right)$ for all $i, j$ with $1 \leqslant i<j \leqslant n-1$.

This follows from the inductive hypothesis.

STEP 2. If $K^{\prime}$ and $G^{\prime}$ are as in Step 1 then there exists homogeneous $u \in K \backslash K^{\prime}$ such that $\exp (u) \neq \exp \left(u_{n-1}\right)$.

Suppose $v \in K \backslash K^{\prime}$ is homogeneous and $\exp (v)=\exp \left(u_{n-1}\right)$. Then $\partial v=\partial u_{n-1}$ so $u=v-l c(v)\left(l c\left(u_{n-1}\right)\right)^{-1} u_{n-1} \in K \backslash K^{\prime}$ is homogeneous with $\exp (u) \prec \exp \left(u_{n-1}\right)$.

STEP 3. There exist $K^{\prime}$ and $\mathcal{G}^{\prime}$ as in Step 1 such that some homogeneous $u \in K \backslash K^{\prime}$ satisfies $\exp \left(u_{n-1}\right) \prec \exp (u)$.

Let $K^{\prime}$ and $G^{\prime}$ be as in Step 1. By Step 2 there exists homogeneous $w \in K \backslash K^{\prime}$ such that $\exp (w) \neq \exp \left(u_{n-1}\right)$. If $\exp (w) \prec \exp \left(u_{n-1}\right)$ set $u=u_{n-1}$ and let $K^{\prime \prime}$ be the linear subspace spanned by $\left\{u_{1}, u_{2}, \ldots, u_{n-2}, w\right\}$. It is easy to $\operatorname{see} \exp \left(w^{\prime}\right) \prec \exp \left(u_{n-1}\right)$ for all $w^{\prime} \in K^{\prime \prime}$. Choose a basis $\mathcal{G}^{\prime \prime}$ as in Step 1 and replace $K^{\prime}$ with $K^{\prime \prime}$ and $\mathcal{G}^{\prime}$ with $\mathcal{G}^{\prime \prime}$.

STEP 4. There is a homogeneous basis $\mathcal{G}=\left\{u_{1}, u_{2}, \ldots, u_{n}\right\}$ of $K$ such that $u_{1} \prec u_{2}$ $\prec \cdots \prec u_{n}$ and $\exp \left(u_{i}\right) \notin \mathcal{N}\left(u_{j}\right)$ for all $i, j$ with $1 \leqslant i<j \leqslant n$.

Let $K^{\prime}, \mathcal{G}^{\prime}$, and $u$ be as in Step 3 and write $u$ as in equation 5 . Set

$$
\mathcal{M}=\mathbb{N} \backslash\left\{\exp \left(u_{i}\right): i=1,2, \ldots, n-1\right\}
$$

and set

$$
u_{n}=u-\sum_{i=1}^{n-1} c_{\exp \left(u_{i}\right)}\left(l c\left(u_{i}\right)\right)^{-1} u_{i} .
$$

Clearly $\exp \left(u_{n-1}\right) \prec \exp \left(u_{n}\right)$ so we only need to show $\mathcal{N}\left(u_{n}\right) \subseteq \mathcal{M}$. Set

$$
v=u-\sum_{i=1}^{n-1} c_{\exp \left(u_{i}\right)} \mathrm{t}^{\exp \left(u_{i}\right)}
$$

and set $v_{i}=u_{i}-l c\left(u_{i}\right) \mathrm{t}^{\exp \left(u_{i}\right)}$ for $i=1,2, \ldots, n-1$. Then $\mathcal{N}(v) \subseteq \mathcal{M}$ by construction and $\mathcal{N}\left(v_{i}\right) \subseteq \mathcal{M}$ for $i=1,2, \ldots, n-1$ by our choice of $\mathcal{G}^{\prime}$. Moreover

$$
u_{n}=v-\sum_{i=1}^{n-1} c_{\exp \left(u_{i}\right)} l c\left(u_{i}\right)^{-1} v_{i}
$$


so

$$
\mathcal{N}\left(u_{n}\right) \subseteq \mathcal{N}(v) \cup\left(\bigcup_{i=1}^{n-1} \mathcal{N}\left(v_{i}\right)\right) \subseteq \mathcal{M}
$$

as desired.

THEOREM 4.5. Let $K$ be a homogeneous linear subspace of $\mathcal{X}_{+}$and let $I$ be the ideal generated by $K$. Then $I=U(\mathcal{X}) K$ and $U(\mathcal{X} / K) \cong U(\mathcal{X}) / I$. Moreover the basis $\mathcal{G}$ of $K$ described in Lemma 4.4 is a Gröbner basis of $I$.

Proof: It is easy to see $\mathcal{X}_{+}$, and hence $K$, contains only normal elements of $U(\mathcal{X})$. The relations $I=U(\mathcal{X}) K$ and $U(\mathcal{X} / K) \cong U(\mathcal{X}) / I$ can be proved using the PBW Theorem (see [2, Thoerem 3.2.2]) and the universal property of the enveloping algebra.

To prove $\mathcal{G}$ is a Gröbner basis of $I$ we show the remainder is 0 when the left division algorithm by $\mathcal{G}$ is applied to $S^{\ell}\left(u_{i}, u_{j}\right)$ (see [7, Theorem 2.1]). Then $\mathcal{G}$ satisfies the so-called "Buchberger S-pair criterion", which is [7, Theorem 3.2].

For each $i, j$ with $1 \leqslant i<j \leqslant n$ it is enough to show that $S^{\ell}\left(u_{i}, u_{j}\right)=v_{1} u_{i}+v_{2} u_{j}$ where

$$
\exp \left(v_{1} u_{i}\right) \preccurlyeq \exp \left(S^{\ell}\left(u_{i}, u_{j}\right)\right), \quad \exp \left(v_{2} u_{j}\right) \preccurlyeq \exp \left(S^{\ell}\left(u_{i}, u_{j}\right)\right)
$$

and for all $\alpha \in \mathcal{N}\left(v_{i}\right)$ there does not exist $\beta \in \mathbb{N}^{p}$ such that $\exp \left(u_{j}\right)+\alpha=\exp \left(u_{i}\right)+\beta$. We may pass to the case $i=1$ and $j=2$ by [7, Proposition 2.11].

Since $u_{1}, u_{2} \in \mathcal{X}_{+}$and $u_{1} \prec u_{2}$ there exist $p_{1}, p_{2} \in\{1,2, \ldots, p\}$ with $p_{1}<p_{2} \leqslant p$ such that $\exp \left(u_{1}\right)=\phi\left(p_{1}\right) e_{p_{1}}$ and $\exp \left(u_{2}\right)=\phi\left(p_{2}\right) e_{p_{2}}$. Thus $\alpha=\phi\left(p_{1}\right) e_{p_{1}}, \beta=\phi\left(p_{2}\right) e_{p_{2}}$, and $\gamma=\alpha+\beta$, which implies $\lambda=l c\left(u_{2}\right)$ and $\mu=l c\left(u_{1}\right) \varepsilon\left(u_{2}, u_{1}\right)$. Set $v_{1}=\varepsilon\left(u_{2}, u_{1}\right)\left(u_{2}\right.$ $\left.-l c\left(u_{2}\right) t_{p_{2}}^{\phi\left(p_{2}\right)}\right)$ and $v_{2}=\varepsilon\left(u_{2}, u_{1}\right)\left(u_{1}-l c\left(u_{1}\right) t_{p_{1}}^{\phi\left(p_{1}\right)}\right)$. Then

$$
\begin{aligned}
S^{\ell}\left(u_{1}, u_{2}\right) & =\lambda \mathrm{t}^{\gamma-\alpha} u_{1}-\mu \mathrm{t}^{\gamma-\beta} u_{2} \\
& =l c\left(u_{2}\right) t_{p_{2}}^{\phi\left(p_{2}\right)} u_{1}-l c\left(u_{1}\right) \varepsilon\left(u_{2}, u_{1}\right) t_{p_{1}}^{\phi\left(p_{1}\right)} u_{2} \\
& =\left(u_{2}+v_{1}\right) u_{1}-\left(\varepsilon\left(u_{2}, u_{1}\right) u_{1}-v_{2}\right) u_{2} \\
& =v_{1} u_{1}+v_{2} u_{2}
\end{aligned}
$$

with $\exp \left(v_{1}\right) \prec \exp \left(u_{2}\right)$ and $\exp \left(v_{2}\right) \prec \exp \left(u_{1}\right)$.

Let $\alpha \in \mathcal{N}\left(u_{2}\right)$ be arbitrarily chosen. Then $\alpha=\phi\left(p_{3}\right) e_{p_{3}}$ for some $p_{3} \in\{1,2, \ldots, p\}$ with $p_{3} \leqslant p_{2}$ since $u_{2} \in \mathcal{X}_{+}$. If there exists $\beta \in \mathbb{N}^{p}$ such that $\exp \left(u_{j}\right)+\alpha=\exp \left(u_{i}\right)+\beta$ then $\phi\left(p_{2}\right) e_{p_{2}}+\phi\left(p_{3}\right) e_{p_{3}}=\phi\left(p_{1}\right) e_{p_{1}}+\beta$. We must have $\phi\left(p_{3}\right) e_{p_{3}}=\phi\left(p_{1}\right) e_{p_{1}}$ and $\beta=\phi\left(p_{2}\right) e_{p_{2}}$ since $p_{1}<p_{2}$. But this implies $\exp \left(u_{1}\right)=\alpha \in \mathcal{N}\left(u_{2}\right)$, which contradicts our choice of basis.

It follows from what we just proved that $\exp \left(v_{1} u_{1}\right) \neq \exp \left(v_{2} u_{2}\right)$. This implies $\exp \left(v_{1} u_{1}\right) \preccurlyeq \exp \left(S^{\ell}\left(u_{1}, u_{2}\right)\right)$ and $\exp \left(v_{2} u_{2}\right) \preccurlyeq \exp \left(S^{\ell}\left(u_{1}, u_{2}\right)\right)$ as desired. 


\section{REFERENCES}

[1] M. Aubry and J.-M. Lemaire, 'Zero divisors in enveloping algebras of graded Lie algebras', J. Pure Appl. Algebra 38 (1985), 159-166.

[2] Yu. A. Bahturin, A.A. Mikhalev, V.M. Petrogradsky and M.V. Zaicev, Infinite dimensional Lie superalgebras (DeGruyter, Berlin, 1992).

[3] E.J. Behr, 'Enveloping algebras of Lie superalgebras', Pacific J. Math. 130 (1987), 9-25.

[4] A.D. Bell, 'A criterion for primeness of enveloping algebras of Lie superalgebras', J. Pure Appl. Algebra 69 (1990), 111-120.

[5] R. Bøgvad, 'Some elementary results on the cohomology of graded Lie algebras', in Homotopie Algébrique et Algèbre Locale (Luminy, 1982), Astérisque 113-114 (Soc. Math. France, Paris, 1984), pp. 156-166.

[6] J.L. Bueso, F.J. Castro-Jiménez, J. Gómez-Torrecillas and F. J. Lobillo, 'Homological computations in PBW modules', Algebr. Represent. Theory 4 (2001), 201-218.

[7] J.L. Bueso, F.J. Castro-Jiménez, J. Gómez-Torrecillas and F. J. Lobillo, 'An introduction to effective calculus in quantum groups', in Rings, Hopf algebras and Brauer Groups, (S. Caenepell and A. Verschoren, Editors), Lecture Notes in Pure and Applied Mathematics 197 (Marcel Dekker, New York, 1998), pp. 55-83.

[8] J.L. Bueso, F.J. Castro-Jiménez, J. Gómez-Torrecillas and F.J. Lobillo, 'Primality test in iterated ore extensions', Comm. Algebra 29 (2001), 1357-1371.

[9] E. Kirkman, J. Kuzmanovich and L. Small, 'Finitistic dimensions of Noetherian rings', J. Algebra 147 (1992), 350-364.

[10] L. Le Bruyn, 'Trace rings of generic 2 by 2 matrices', Mem. Amer. Math. Soc. 66 (1987).

[11] J.C. McConnell and J.C. Robson, Noncommutative Noetherian rings (Wiley, Chichester, England, 1987).

[12] K.L. Price, 'Homological properties of color Lie super algebras', in Advances in Ring Theory (Granville, OH, 1996), (S.K. Jain and S. Tariq Rizvi, Editors) (Birkhäuser, 1997), pp. 287-293.

[13] K.L. Price, 'Primeness criterion for universal enveloping algebras of Lie color algebras', J. Algebra 235 (2001), 589-607.

Department of Mathematics

University of Wisconsin Oshkosh

Oshkosh, WI 54901-8631

United States of America

e-mail: pricek@uwosh.edu 